\title{
RECONFIGURATION: A TOOL FOR DESIGNING NEW SHIPS
}

By:

K.R. Davey

R.E. Hebner

IEEE Electric Ship Technologies Symposium, Philadelphia, Pennsylvania, Jul 25-27, 2005

PN - 295

Center for Electromechanics

The University of Texas at Austin

PRC, Mail Code R7000

Austin, TX 78712

(512) $471-4496$ 


\title{
Reconfiguration: A Tool for Designing New Ships
}

\author{
Kent R. Davey, Fellow, IEEE and Robert E. Hebner, Fellow, IEEE
}

\begin{abstract}
The objective of reconfiguration is to re-route power during failure or compromised conditions to improve fight through capability and survivability. Rapid algorithms for the task may serve the dual purpose of managing power flow under dynamic load change. Among the critical constraints of power redirection is the line current rating. Reconfiguration algorithms serve an equally useful task in system grid design. An exhaustive search optimization routine can be used to specify grid layout necessary to guarantee $100 \%$ performance in the face of single and multiple line loss. A genetic optimization routine is employed to determine required line rating and switch configuration during generator loss to satisfy critical load demands. The combination of these tests results in line current rating and switch locations necessary for reliable ship operation for the least cost.
\end{abstract}

Index Terms-binary, genetic, optimization, planning, reconfiguration.

\section{INTRODUCTION}

$\mathrm{R}$ ESEARCHERS characteristically view reconfiguration as a method for handling emergency conditions, i.e., a set of procedures to mitigate a compromised state during combat or component failure. The authors submit that a significant benefit is realized by executing a global reconfiguration continuously, since it maximizes system resources, resources that are necessarily changing dynamically. The reconfiguration algorithm must be fast to accomplish the task, and it is achieved by replacing distributed electrical loads with equivalent dynamic impedances [1]. Subspace optimization tools [2] and binary integer optimization algorithms [3] have proved useful in solving this system. The subspace approaches have the benefit of attacking the problem in smaller portions, optimizing a subset of the problem and communicating that solution to its neighbors.

Much of this work might be viewed as having only retrograde significance; once the system is constructed, the reconfiguration algorithm gives direction about how to change

The work was supported by The Office of Naval Research.

K. R. Davey is with the Center for Electromechanics at the University of Texas, 10100 Burnet Rd, Bldg EME 133, Austin, TX 78758 USA (e-mail: k.davey@mail.utexas.edu).

R. E. Hebner is with the Center for Electromechanics at the University of Texas, 10100 Burnet Rd, Bldg EME 133, Austin, TX 78758 USA (e-mail: r.hebner@mail.utexas.edu). it for optimal performance. In addition, there is a very useful function of the reconfiguration algorithm that gives ship designers specific instructions on the layout of the electrical system a priori. Consider the task of choosing cable sizing; the common design approach is to compute the line load and add $20-50 \%$. In reality, the designer should be asking what the cables should look like to guarantee $100 \%$ operation to the remaining system after reconfiguration. The idea is to increase the cable ratings to accommodate power flow rerouting. Quantifying the new ratings is not at all intuitive, and using the standard $20-50 \%$ rule will miss the mark by a significant amount in both directions.

A similar problem arises when a generator is compromised and power must be routed from the remaining generators to supply critical loads. This task can only be performed under the following conditions:

1. The grid is fully interconnected. The greater the interconnection, the greater the possibility of finding useful reconfigurations under compromised conditions.

2. The loads can be approximated in real time and replaced with equivalent impedances.

3. The loads can be isolated to the exclusion of their feed lines.

This paper concentrates on a way to use reconfiguration to predict line ratings throughout a ship, and to specify where isolation switches are required.

\section{Specifying CurRent Ratings for Single Line Loss}

Consider the grid system shown in Fig. 1. Note that each trunk line of the grid has switches on either end, the state of which are determined by optimization analysis. The parallel loads $Z_{p}$ represent a number of tapped parallel loads over the length of the trunk line. A reconfiguration algorithm was executed sequentially, compromising each of the 24 series segments, one at a time. The process can be repeated for a multiple line loss. The three red loads have mandatory minimal power requirements for all cases. The reconfiguration seeks the best switch configuration, rerouting power throughout the grid to meet those required minimums. The current rating on all lines was recorded for each case, while maximizing power flow and minimizing loss to the remainder of the system. 


$$
\mathfrak{I}=\sum I_{s}^{2} R_{s}-\sum I_{p}^{2} Z_{p}
$$

subj

$$
\begin{aligned}
& I_{P} \geq I_{P_{-} \min } \\
& \text { Gen }_{\text {Load }} \leq P_{\text {Rated }}
\end{aligned}
$$

$\mathrm{R}_{\mathrm{s}}$ and $\mathrm{Z}_{\mathrm{p}}$ are the series transmission resistance and parallel load impedance respectively. $\mathrm{P}_{\text {Rated }}$ is the rated power for the generators. No restriction is placed on the trunk line series current $I_{s}$. The parallel currents $I_{p}$ are monitored to ensure that a minimum power be available for critical loads, as is the maximum power delivered by the generators. An exhaustive search is performed over the switch solution space for any one line failure. The switch configuration which realizes the lowest index and does not violate the two constraint conditions is recorded; all series line currents $I_{S}{ }^{\prime}$ for the best configuration are also recorded. The procedure is repeated for the next line to be compromised, obtaining a set of currents $\mathrm{I}_{\mathrm{s}}{ }^{\prime \prime}$. The series line requirement is the maximum of the two vectors $\mathrm{I}_{\mathrm{s}}{ }^{\prime}$ and $\mathrm{I}_{\mathrm{s}}$ " on a component by component basis. The process is repeated sequentially compromising all series lines, and keeping the best current after each step as $\mathrm{I}_{\mathrm{s}}{ }^{\prime}=\max \left(\mathrm{I}_{\mathrm{s}}{ }^{\prime}\right.$, $\left.\operatorname{abs}\left(\mathrm{I}_{\mathrm{s}}^{\prime \prime}\right)\right)$.

The upper trace in Fig. 1 shows the maximum of the current obtained for each line, while the lower trace shows the normal operation performance. A minimum critical load is demanded on the three highlighted loads in Fig. 1. It is obvious that some lines serve a critical role in rerouting power. The reconfiguration algorithm quantifies that effect, dictating the specifications required to guarantee fight through capability with a single line failure.
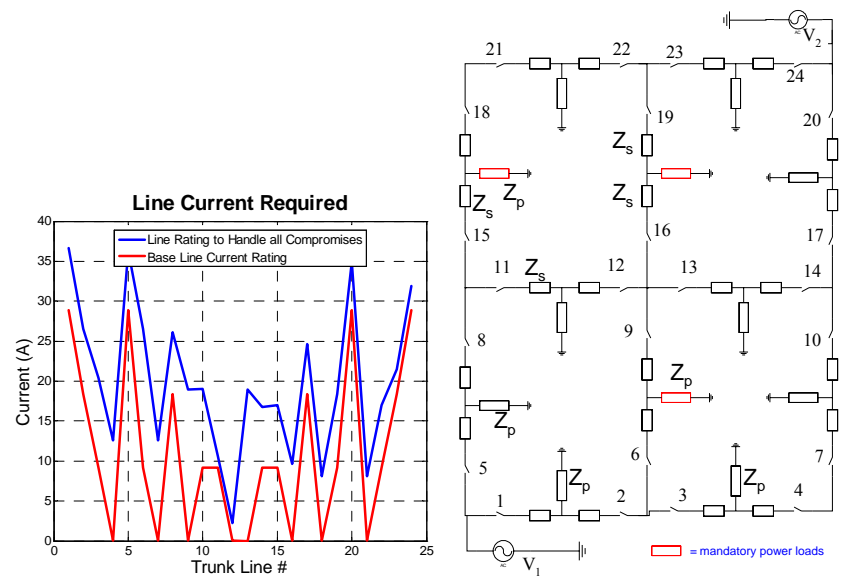

Fig. 1 Line rating changes determined from running reconfiguration on a small grid.

\section{SPECIFYING CURRENT RATINGS FOR A DOUBLE LiNE LOSS}

The problem grows rapidly as the complexity of the compromise increases. With a double line failure and $\mathrm{n}$ lines, the number of failure pairs is $\Sigma(n-1)$, or 276 for a 23 switch system. Fig. 2 shows the results when an exhaustive search optimization is applied to the grid in Fig. 1. As expected intuitively, the current that each trunk line must be capable of handling increases on every line. But the increase is not as expected. Fig. 3 shows the ratio of the required current between the two cases. Having the ability to sustain a two line failure and $100 \%$ fight through capability places a high demand on one segment relatively unused, but most lines increase by a modest index less than two. Segment 12 becomes important for distributing power during a double line failure, but not for a single.

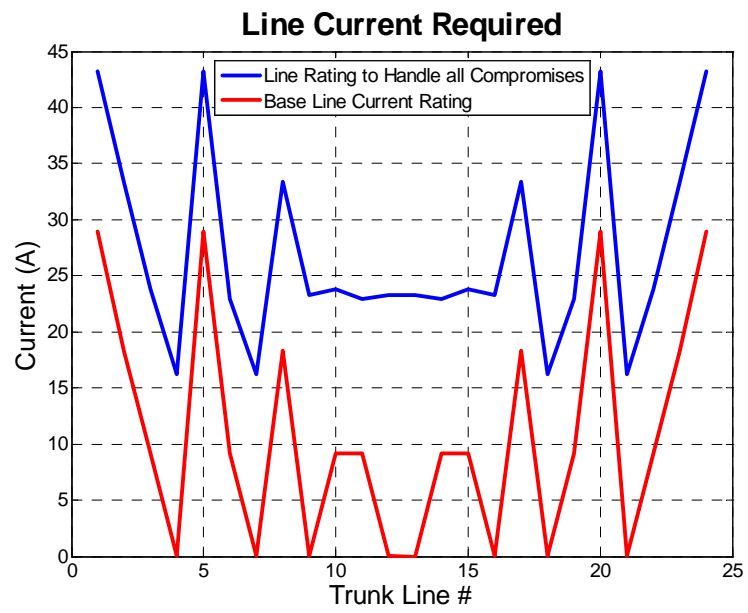

Fig. 2 Line ratings with two lines compromised.

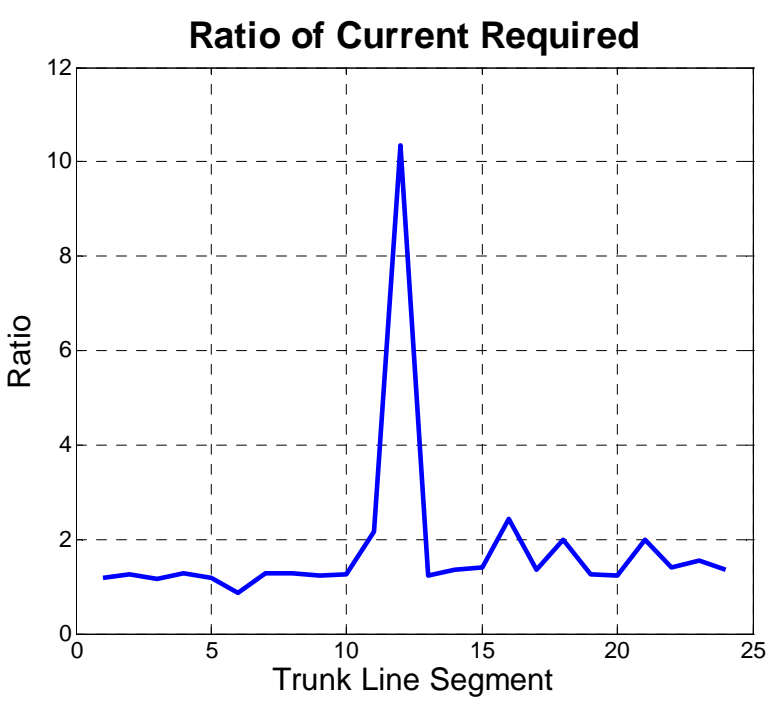

Fig. 3 Ratio of current required on every trunk segment for a one and two line failure fight through capability.

\section{MAINTAINING CRITICAL LOADS With GENERATOR LoSS}

A second planning question is "where should switches be placed?" Fig. 1 shows 24 switches placed on either end of every transmission segment. The segment has multiple loads it handles. If a fault occurs, it is most desirable to isolate only the compromised load. If the internal protection of that component fails, the next desirable option is to remove both 
ends of the trunk segment feeding the anomalous load. The 24 switches are justified for this protection.

Consider the added complication of reconfiguration with a generator loss. The available power generation capability becomes less than the system load demand. Slowing of remaining generators will be commensurate with asynchronous operation unless load is shed quickly. A first reaction might be that additional switches are required in line with the parallel loads as shown in Fig. 4. For this test system, 12 additional switches are shown.

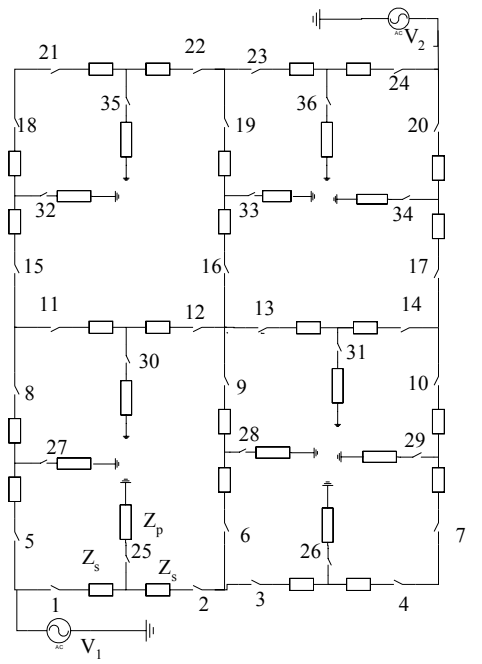

Fig. 4 Additional switches required to handle the loss of one or more generators.

The exhaustive search for one line loss requires $74 \mathrm{~s}$ to run on a $3 \mathrm{GHz}$ Pentium IV computer with only 24 switches. Since the number of unknowns scales as $2^{\mathrm{n}}$, this corresponds to 16.7 million options. Some are eliminated automatically when they isolate key loads. The two line outage requires 10 hours to run using the direct exhaustive search. The number of possibilities for the 36 switch system is $6.87 \cdot 10^{10}$. Are switches 25 through 36 necessary? Fig. 5 shows the result of reconfiguring the 24 switch system when generator \#2's capability is cut in half. The optimization solution feeds two of the three critical loads with generator \#1, while cutting the grids so that generator \#2 feeds only three loads. Generator 1 must be slightly overrated to do this without parallel load switches. There is no solution when generator \#2 is taken off line without the ability to shed load through the additional switches in Fig. 4.

The optimization is so time consuming that other solution methods are now required. Before discussing the results of generator loss analysis and its implications for grid planning, a brief discussion of the optimization found most helpful is in order.

\section{A. Genetic Algorithm and Pattern Search Optimization}

Binary optimization problems are especially difficult since no gradient information is available for the search. Two direct (no gradient) optimization procedures were investigated, pattern search [4] [5] and genetic algorithm [6]. Both have in common the integration of sub-patterns to find the global minima. The former attempts this by building an $\mathrm{n}$ dimensional grid which grows and then shrinks as the algorithm advances. The algorithm investigates the optimization index about a point in $\mathrm{n}$ different directions; the magnitude of these search vectors changes as the algorithm progresses. This algorithm proved to be less effective than the genetic algorithm.

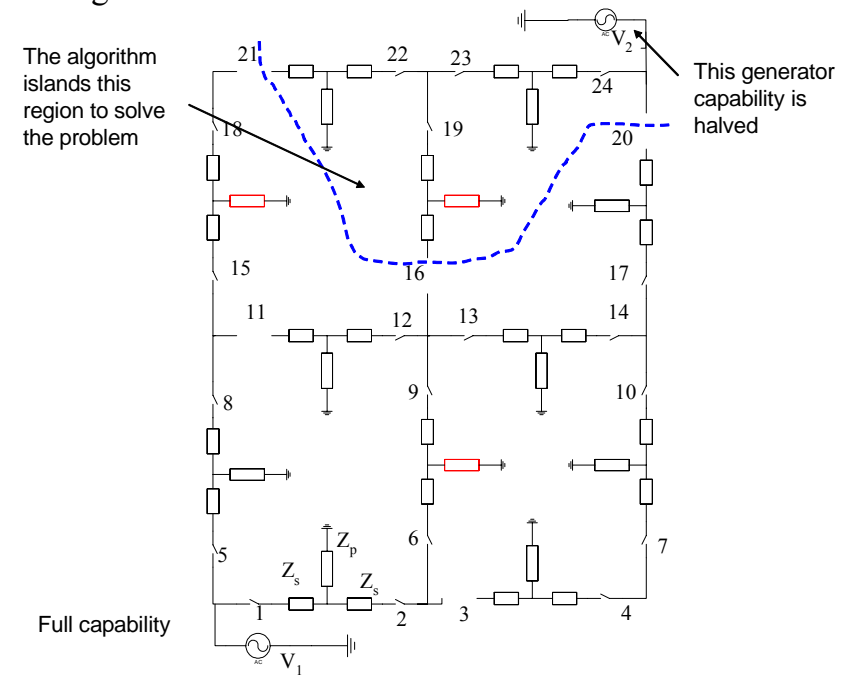

Fig. 5 Results of optimizing a system when generator 2's capability is cut in half.

The genetic algorithm is a more mature search tool. It begins with multiple starting guesses, referred to as the population. New solutions called children are constructed by intermixing subsets of these starting guesses. In addition to intermixing, a subpopulation of the children's solution set is randomly varied. This perturbation is called mutation. The mutation rate, i.e., the frequency for which random changes are introduced, decreases as the algorithm progresses. Each new solution set is called a generation. The number of generations greatly affects the speed of the algorithm.

Constraints are imposed by using penalty functions. The condition in (1) is written as

$$
\mathfrak{I}=\frac{\sum I_{s}{ }^{2} R_{s}-\sum I_{p}{ }^{2} Z_{p}}{P_{\text {Rated Total }}}+\left(I_{P}<I_{P_{-} \min }\right)+\left(\text { Gen }_{\text {Load }}>P_{\text {Rated }}\right)
$$

In this form the constraints are logical functions. Every load current that is less than the minimum adds a one to the index. Every generator that exceeds its rating also adds a one to the index. Notice that resistive dissipation minus useful power is normalized so that the constraints become consequential. Fig. 6 shows how the computation time and the solution error change with the number of generations, assuming an initial population of 20 for the grid in Fig. 1. The solution switch set is compared to that achieved by the exhaustive search algorithm. Excellent results are obtained in $2 \%$ of the time required by the exhaustive search at 60 generations. The error is predicted by comparing the index achieved to the best possible. The ordinate in the lower graph 
represents the ratio of time required by the genetic algorithm to that required in the exhaustive search.
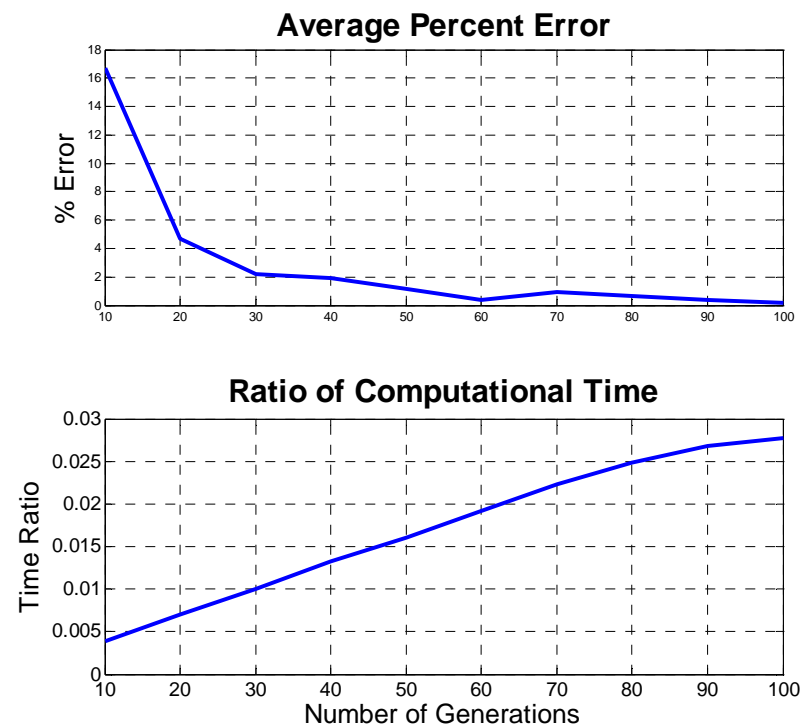

Fig. 6 Genetic algorithm optimization as a function of the number of generations.

Note that the time ratio does not grow linearly with generation count. This happens because a stall limit is built into the algorithm. The algorithm stops when further progress is not achieved after an established time ( $20 \mathrm{~s})$. The results do not show an asymptotic decrease with generation count because of the random nature of the search. The Monte Carlo aspect of the algorithm results in some solution variation.

\section{B. Optimization Results with Generator Loss}

Having the additional ability to isolate loads yields the greatest flexibility for reconfiguration, but the system becomes more expensive to construct and maintain. The impedance $Z_{p}$ represents all the loads in a trunk segment including parasitic capacitance to ground. In most cases, the one switch shown for each parallel load would likely be implemented with multiple switches because of proximity. Only a few parallel switches are required to handle generator failures.

The solution shown in Fig. 7 shows what the genetic algorithm determines as optimal configurations for the test grid when generators 1 and 2 fail. In keeping with intuition, the critical loads are separated from the remaining grid in each case, i.e., smaller islands are formed which the limited generation capability can handle. Of more interest is the shedding of load within the smaller surfaced island. This analysis suggests that it is necessary to place parallel load switches on only two locations to handle either generator failure, i.e., on loads 30 and 35 . Commensurate with these solutions are line currents which exceed normal steady state values.

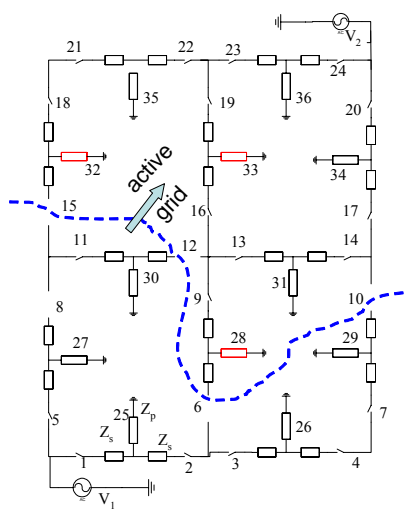

(a) Switch configuration when Generator 1 (lower left) fails

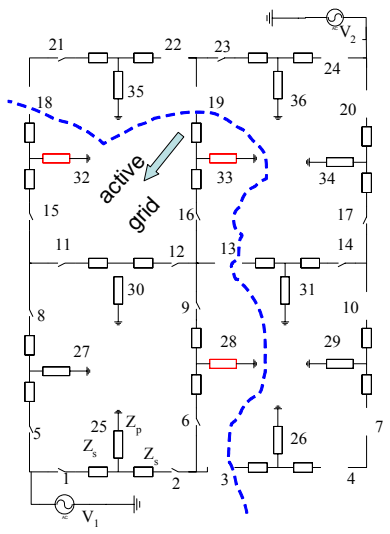

(b) Switch configuration when Generator 2 (upper right) fails
Fig. 7 Optimized switch configuration to meet critical loads when generators fail.

\section{CONCLUSIONS}

Reconfiguration algorithms are a critical key to intelligent planning in electric ship design. They allow the planner to quantitatively address questions such as "What is the correct current capacity for the distribution feeders enabling $100 \%$ fight through operability?" It also addresses questions about where switches should be placed to ensure continued power delivery to critical loads during generator failure. The size of these optimization problems becomes unmanageable quickly with direct search methods. Genetic and pattern search techniques which mix and match groups of useful switch orders prove effective for obtaining an answer quickly, albeit not necessarily the best answer. Reconfiguration testing quantifies the line carrying ability systematically. Once designed, the reconfiguration will also indicate how load current should be redirected to take advantage of lower impedance feeders.

\section{REFERENCES}

[1] K. Davey, "Fault Analysis on a Naval Power Grid using Equivalent Impedances," IASME Transactions, Issue 2, Vol. 1, April 2004, pp. 247252.

[2] C. Meek, K. Davey, D. Tucker, B. Shutt, and R. Hebner, "Approaches to Shipboard Power Management," ASNE Reconfiguration and Survivability Symposium 2005, Jacksonville FL, Feb. 16-17, 2005, paper session III-1.

[3] K. Davey and R. Hebner, "Reconfiguration of Shipboard Power Systems," IASME Transactions, Issue 2, Vol. 1, April 2004, pp. 241-246.

[4] R. M. Lewis and V. Torczon, "Pattern Search Methods for Linearly Constrained Minimization," SIAM Journal on Optimization, Vol. 10, Number 3, 2000, pp. 917-941.

[5] C. Audet and J. E. Dennis Jr., "Analysis of Generalized Pattern Searches," SIAM Journal on Optimization, Vol. 13, Number 3, 2003, pp. 889-903.

[6] D. E. Goldberg, "Genetic Algorithms in Search," Optimzation \& Machine Learning, Addison-Wesley, 1989. 


\section{BIOGRAPHIES}

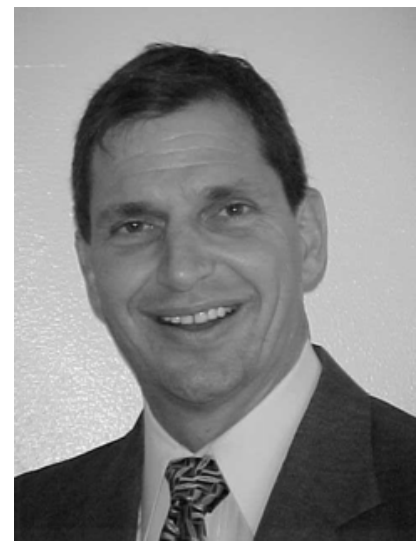

Kent Davey became a Member of IEEE in 1980, a Senior Member in 1986, and a Fellow in 2004. He was born in New Orleans, Louisiana in 1952. He received his BS in EE from Tulane in 1974, his MS in Power Engineering from Carnegie Mellon in 1976, and his MS in physics from the University of Pittsburgh in 1976. He received his $\mathrm{PhD}$ in $\mathrm{EE}$ from the Massachusetts Institute of Technology in 1980. He completed a Fulbright in Finland on atmospheric physics in 1981.

From 1974 to 1976 he worked at Westinghouse as a large turbine generator designer. From 1979 to 1980 he served as an assistant professor at Texas A\&M University. From 1980 to 1994 he served as a tenured associate professor at the Georgia Institute of Technology. From 1994 to 2002, he served as technical director of American Maglev Technology in Edgewater, Florida. He presently serves as a research scientist at the Center for Electromechanics at the University of Texas in Austin, Texas. Dr. Davey has research activity in electromechanical machine design, pulsed electromechanical devices, HTS Trapped Field magnets, electroporation, and magnetic stimulation of biological tissue.

Dr. Davey is a Fellow with IEEE and presently serves as editor of IEEE Transactions on Magnetics. He is actively involved in the review of conference and journal papers for various IEEE activities.1943.

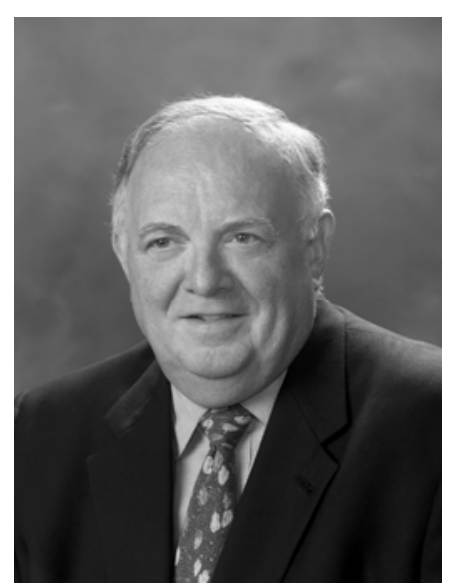

Robert E. Hebner, Ph.D., is Director of the Center for Electromechanics at the University of Texas at Austin. The Center develops technology, primarily novel motors, generators, and suspension components, and teams with companies to get the technology into the market.

Previously, Dr. Hebner was the acting Director of the U.S. National Institute of Standards and Technology (NIST). In addition, he has directed NIST's Electronic and Electrical Engineering Laboratory, a laboratory with a staff of more than 250. He also worked at the Defense Advanced Research Projects Agency where he developed programs to improve semiconductor manufacturing.

Throughout his career, Dr. Hebner has been active in having authored or coauthored more than one hundred technical papers and reports. He has extensive experience in international technology programs. This work included the modernization of the measurement systems needed to support global trade and the assessment of the effectiveness of government technology programs in stimulating domestic economies. 